\title{
Quantum States with Maximum Information Entropy. I.
}

\author{
W. Bayer and W. Ochs \\ Sektion Physik der Universität München
}

(Z. Naturforsch. 28 a, 693-701 [1973] ; received 23 January 1973)

\begin{abstract}
In the frame of the information theory approach to quantum statistics, we examine the conditions which a given information " $\operatorname{Tr}(W A)=m$ " must satisfy in order to determine a unique quantum state with maximum information entropy.
\end{abstract}

\section{Introduction}

In the last fifteen years the information theory approach has led to an original foundation of statistical thermodynamics ${ }^{1-4}$. This approach is founded on the general principle of inductive reasoning that, to make statistical inferences on the basis of incomplete information one must use the most unbiased probability assumptions compatible with the given information. By means of the information entropy as a measure of the "missing information" (or "amount of uncertainty"), Jaynes ${ }^{1}$ first has formulated this principle precisely and has applied it to statistical physics:

Principle of Maximum Entropy: If only partial information is given about the exact state (microstate) of a physical system, then the system must be described by that macrostate (statistical ensemble of microstates) which has the maximum information entropy compatible with the given information.

This formulation indicates the wide range of applicability of the principle of maximum entropy. In order to apply this principle, however, in the frame of a specific physical theory, one first needs the precise definition of the macrostates of the theory in question and a concrete measure of the information entropy of these macrostates.

The application of the principle of maximum entropy in the frame of quantum theory poses no conceptual difficulties. In a systematic development of quantum mechanics, all states of a quantummechanical (q.m.) system may be characterized ${ }^{5-7}$ as mean value functionals $M$ on the set $\mathscr{B}(\mathscr{H})$ of all bounded, self-adjoint (s.a.) operators on the Hilbert space $\mathscr{H}$ of the system satisfying the properties

1) $M(1)=1, M\left(A^{2}\right) \geqq 0$,

Reprint requests to Dr. W. Ochs, Sektion Physik, Lehrstuhl Prof. Süßmann, D-8000 München 2, Theresienstr. 37, Germany.
2) $M(A+B)=M(A)+M(B)$,

$3)$ if $\left\{P_{i}: i \in \mathbf{N}\right\}$ is a decreasing sequence of projection operators with $\stackrel{\infty}{\Lambda} P_{i}=0$, then

$$
\lim _{i \rightarrow \infty} M\left(P_{i}\right)=0 .
$$

There exists a 1-1-correspondence between the set of all functionals $M: \mathscr{B}(\mathscr{H}) \mapsto \mathrm{R}$ with the properties (1.1) to (1.3) and the set $\mathfrak{W}$ of all positive, s.a. operators of trace one (state operators). Every mean value functional $M$ can be represented, by means of the corresponding state operator $W$, in the form

$$
M(A)=\operatorname{Tr}(W A) \equiv \sum_{i=1}^{\infty}\left(\varphi_{i}, W A \varphi_{i}\right)
$$

where $\left\{\varphi_{i}: i \in \mathbf{N}\right\}$ is an arbitrary complete orthonormal system (basis) of $\mathscr{H}^{5-7}$. The definition of the trace in (1.4) is reasonable since the relation

$$
\begin{aligned}
\sum_{i=1}^{\infty}\left(\varphi_{i}, W A \varphi_{i}\right) & =\sum_{i=1}^{\infty}\left(\varphi_{i}, A W \varphi_{i}\right) \\
& =\int_{-\infty}^{\infty} \lambda \mathrm{d} \operatorname{Tr}\left[W E_{A}(\lambda)\right],
\end{aligned}
$$

where $A=\int_{-\infty}^{\infty} \lambda \mathrm{d} E_{A}(\lambda)$ is the spectral representation of $A$, holds true for all $A \in \mathscr{B}(\mathscr{H}), W \in \mathfrak{W}$ and for all bases $\left\{\varphi_{i}: i \in \mathbf{N}\right\}$ of $\mathscr{H}$. On the other hand, the statements of (1.5) are not valid for arbitrary s.a. operators $A$. In case of an unbounded $A$, the sums in (1.5) are in general not defined; and, even if they are defined, they are not necessarily independent of the basis chosen ${ }^{7}$. Hence if unbounded operators must also be considered - and this will turn out to be indispensable in our problem - then we must generalize the above mean value functional. Equation (1.5) suggests the expression

$$
\tau(W A) \equiv \tau(A W) \equiv \int_{-\infty}^{\infty} \lambda \mathrm{d} \operatorname{Tr}\left[W E_{A}(\lambda)\right]
$$


as the generalized mean value functional for arbitrary s.a. operators. Obviously, $\tau(W A)$ has a unique (not necessary finite) value if and only if at least one of the two integrals

$$
\begin{aligned}
& \tau\left(W A^{+}\right)=\int_{0}^{\infty} \lambda \mathrm{d} \operatorname{Tr}\left[W E_{A}\left(\lambda_{-}\right)\right], \\
& \tau\left(W A^{-}\right)=\int_{-\infty}^{0}\left(-\lambda_{-}\right) \mathrm{d} \operatorname{Tr}\left[W E_{A}\left(\lambda_{)}\right)\right]
\end{aligned}
$$

is finite. In the particular case of a pure point spectrum of $A$ with the spectral representation $A=\sum_{n \in G} a_{n} P_{a_{n}}$, we have

$$
\tau(W A)=\sum_{n \in G} a_{n} \operatorname{Tr}\left(W P_{a_{n}}\right) .
$$

From definition (1.6) one immediately infers the properties

$$
(\forall c \in \mathrm{R}) \tau(W c A)=c \tau(W A)
$$

and $\quad \tau\left[\left(V_{1}+V_{2}\right) A\right]=\tau\left(V_{1} A\right)+\tau\left(V_{2} A\right)$

for all s.a. operators $V$ of trace class. The additivity of $\tau(W \cdot)$ in $A$ however, is suspected to hold only under restricting conditions ${ }^{7}, \tau(W \cdot)$ is certainly a generalization of the functional $\operatorname{Tr}(W \cdot)$ since $\tau(W A)$ is equal to $\operatorname{Tr}(W A)$ or $\operatorname{Tr}(A W)$ whenever one of the latter is well-defined. Moreover, Langerholc ${ }^{7}$ has shown that $\tau(W \cdot)$ is the only reasonable generalization of the mean value functional $\operatorname{Tr}(W \cdot)$. For this reason we will drop the symbol $\tau(W A)$ and use instead the more familiar notation $\operatorname{Tr}(W A)$ with the generalized meaning implicit.

Partial information about the state of a q.m. system can be formulated in different ways, e.g. "the probability of the result $\alpha_{0}$ in an experiment $E$ is $p_{0}$ " or "the value of the observable $8 A$ is found with certainty in the interval $[a, b]$ " or "the mean value $M(B)$ of the observable $B$ is $\mu$ ". But one easily realizes that any finite amount of such experimentally accessible data can be put in the form

$$
M\left(A_{r}\right)=\operatorname{Tr}\left(W A_{r}\right)=m_{r} ; \quad r=1, \ldots, h
$$

with given real numbers $m_{r}$.

The information entropy $H(W)$ of a quantum state $^{8} W$ has, according to Jaynes ${ }^{1}$ and $\mathrm{Fano}^{9}$, the value $^{10}$

$$
H(W) \equiv-\operatorname{Tr}(W \ln W) .
$$

For a justification of this formula we refer to $9,12,13$. Other authors have also considered a different concept of information entropy in quantum statistics ${ }^{2}, 14$ which characterizes the uncertainty of the outcome of a measurement of a certain observable $A$ for a given quantum state ${ }^{15}$. But this observabledependent entropy concept seems inappropriate for our problem, especially in the case when the given information includes the mean values of several incompatible observables.

In replacing the general notions of the principle of maximum entropy by the corresponding special terms of quantum mechanics we obtain the

Principle of Maximum Entropy for Quantum Statistics: If Eq. (1.10) provides the only information about the state of a q.m. system, then the system must be described by the state operator which has the maximum information entropy $H$ subject to the conditions (1.10).

Though this principle has been applied succesfully to many problems of quantum statistics, the exact conditions under which the information (1.10) determines a unique quantum state with maximum information entropy (QME) have not yet been fully explored. The case of a finite dimensional Hilbert space has been exhaustively studied by Wichmann ${ }^{16}$. The existence of QMEs in infinite dimensional Hilbert spaces was first shown, under special assumptions, by v. Neumann ${ }^{5}$ in deriving the state operator of the canonical ensemble; but a detailed analysis of the conditions of existence of QMEs in separable Hilbert spaces has been made, as far as we know, only for the case $h=1$ by Ingarden and Urbanik $^{2}$. These authors, however, used the observable-dependent entropy concept mentioned above and assumed that $A_{1}$ in (1.10) has a pure point spectrum.

In a series of papers we will examine the conditions which the given information (1.10) must satisfy in order that QMEs exist in an infinite dimensional, separable Hilbert space. In the present paper we reconsider the case $h=1$ of Eq. (1.10), i.e. we assume that the information is given in the form

$$
\operatorname{Tr}(W A)=m,
$$

whereas the case of an arbitrary finite number of given mean values $M\left(A_{r}\right)$ will be treated in a subsequent paper. As for the case $h=1$, the problem can be solved completely and the results of Ingarden and Urbanik are preserved though we use a different entropy concept and make no assumptions about the spectrum of $A$ in (1.12). 


\section{The Case $h=1$}

We first introduce our notations and compile some results from the literature which are needed in the following. Notations: The spectrum of an operator $A$ is denoted by $\sigma(A)$. The supremum [infimum] of the real, closed spectrum of a s.a. operator is denoted by $\bar{\sigma}(A)[\sigma(A)]$. The set of all elements of $\sigma(A)$ except for the isolated eigenvalues with finite multiplicity represents the essential spectrum of $A$ and is denoted by $\sigma_{\mathrm{ess}}(A)$. $A$ complete orthonormal system of eigenvectors of a s.a. operator with a pure point spectrum is called an $A$-basis. To every $A$-basis, there corresponds a $d i$ agonal representation, $A=\sum_{i=1}^{\infty} a_{i} P\left(\varphi_{i}\right)$, where $P\left(\varphi_{i}\right)$ is the projection operator upon the one-dimensional subspace generated by $\varphi_{i}$. The spectral set $S(A)$ of a s.a. operator with a pure point spectrum is the set $\left\{a_{i}: i \in \mathrm{N}\right\}$ of eigenvalues of $A$ which occur in a diagonal representation. $S(A)$ is independent of the particular diagonal representation chosen and contains every eigenvalue $a_{i}$ of $A$ exactly as often as indicated by its multiplicity. We further introduce the subsets

$$
\mathfrak{S}_{A} m \equiv\{W \in \mathfrak{W}: \operatorname{Tr}(W A)=m\}
$$

and

$$
\tilde{\mathfrak{S}}_{A} m \equiv\left\{W \in \mathfrak{S}_{A} m:[W, A]=0\right\} .
$$

Lemma $1^{5}$. a) If $\left\{\varphi_{i}: i \in \mathbf{N}\right\}$ is an arbitrary basis of $\mathscr{H}$, then for all $W \in \mathfrak{W}$ the operator

$$
\tilde{W} \equiv \sum_{i=1}^{\infty} P\left(\varphi_{i}\right) W P\left(\varphi_{i}\right)=\sum_{i=1}^{\infty}\left(\varphi_{i}, W \varphi_{i}\right) P\left(\varphi_{i}\right)
$$

is also contained in $\mathfrak{W}$ and satisfies the relation

$$
H(W) \neq \infty, W \neq \tilde{W} \Leftrightarrow H(W)<H(\tilde{W}) .
$$

b) If $\left\{\varphi_{i}: i \in \mathrm{N}\right\}$ is an $A$-basis, then we find in addition for all $W \in \mathfrak{W}, \operatorname{Tr}(W A)=\operatorname{Tr}(\tilde{W} A)$.

Corollary 2. Let $A$ be a s.a. operator with a pure point spectrum. Then:

a) $\sup \left\{H(W): W \in \mathfrak{S}_{A} m\right\}$

$$
=\sup \left\{H(W): W \in \tilde{\mathfrak{W}}_{A}{ }^{m}\right\} .
$$

b) If $\sup \left\{H(W): W \in \mathfrak{S}_{A}{ }^{m}\right\}<\infty$, then all $W$ for which the supremum is assumed are elements of $\tilde{\mathfrak{S}}_{A}{ }^{m}$.

c) If $\sup \left\{H(W): W \in \mathfrak{S}_{A} m\right\}=\infty$ and if $H(W)$ is finite on $\tilde{\mathfrak{W}}_{A}{ }^{m}$, then $H$ is finite for all elements of $\mathfrak{W}_{A}{ }^{m}$.
Lemma $3^{2}$. 1) Let $\alpha=\left\{a_{1}, a_{2}, \ldots\right\}$ be a sequence of real numbers, let $m$ be a real number with $\inf \alpha<m<\sup \alpha$ and let $\Phi_{\alpha} m$ be the set of all sequences $\varphi=\left\{p_{1}, p_{2}, \ldots\right\}$ of real numbers with the properties

$$
p_{i} \geqq 0, \quad \sum_{i=1}^{\infty} p_{i}=1, \quad \sum_{i=1}^{\infty} a_{i} p_{i}=m .
$$

By introducing

one then finds

$$
H(\varphi) \equiv \sum_{i=1} p_{i}\left|\ln p_{i}\right|
$$

$\sup \left\{H(\varphi): \varphi \in \Phi_{\alpha}{ }^{m}\right\}<\infty \Leftrightarrow(\exists \beta \in \mathrm{R}) \sum_{i=1}^{\infty} e^{-\beta a_{i}}<\infty$.

2) If the sequence $\alpha$ has a finite maximum (or minimum) $a_{\mathrm{s}}$ which occurs in $\alpha$ with the multiplicity $n_{\mathrm{s}}$ then

$$
\sup \left\{H(\varphi): \varphi \in \Phi_{\alpha}^{a_{\mathrm{s}}}\right\}=\ln n_{\mathrm{s}} .
$$

Definition 1. An operator $A$ is called thermodynamically regular (or simply regular) if $A$ is s.a. and if a real number $\beta$ exists with the property

$$
\operatorname{Tr}\{\exp (-\beta A)\}<\infty \text {. }
$$

From Definition 1 we conclude five characteristics of regular operators which represent important necessary conditions for the regularity of a s.a. operator.

Lemma 4. If $A$ is a regular operator, then

$A$ has a pure point spectrum,

$\sigma(A)$ contains only eigenvalues of finite multiplicity,

$\sigma(A)$ has no finite limit point,

$\sigma(A)$ is unbounded, and

$\sigma(A)$ is bounded from one side.

Proof. Since $A$ is s.a. and $\exp \{-\beta A\}$ is of trace class, we find for an arbitrary basis $\left\{\varphi_{i}: i \in \mathrm{N}\right\}$ of $\mathscr{H}$

$$
\begin{aligned}
\operatorname{Tr}\left(e^{-\beta A}\right) & =\sum_{i=1}^{\infty}\left(\varphi_{i}, e^{-\beta A} \varphi_{i}\right) \\
& =\sum_{i=1}^{\infty} \int e^{-\beta \lambda} \mathrm{d}\left(\varphi_{i}, E_{A}(\lambda) \varphi_{i}\right)
\end{aligned}
$$

where $A=\int \lambda \mathrm{d} E_{A}(\lambda)$ is the spectral representation of $A$. If we now consider the projection operator $Q \equiv E_{A}(z)-E_{A}(y)$ for arbitrary $y, z \in \mathrm{R}, y<z$, then we obtain 


$$
\begin{aligned}
\int_{-\infty}^{\infty} \mathrm{e}^{-\beta \lambda} \mathrm{d}\left(\varphi_{i}, E_{A}\left(\lambda_{)}\right) \varphi_{i}\right) & \geqq \int_{y}^{z} e^{-\beta \lambda} d\left(\varphi_{i}, E_{A}(\lambda) \varphi_{i}\right) \\
& \geqq \min \left(e^{-\beta y}, e^{-\beta z}\right)\left(\varphi_{i}, Q \varphi_{i}\right)
\end{aligned}
$$$$
\text { and (2.7) yields }
$$

$$
\begin{aligned}
\operatorname{Tr}\left(e^{-\beta A}\right) & \geqq \sum_{i=1}^{\infty} \min \left(e^{-\beta y}, e^{-\beta z}\right)\left(\varphi_{i}, Q \varphi_{i}\right) \\
& =\min \left(e^{-\beta y}, e^{-\beta z}\right) \operatorname{Tr}(Q) .
\end{aligned}
$$

Hence, the regularity of $A$ implies that all projection operators $E_{A}(z)-E_{A}(y)$ with $-\infty<y<$ $z<\infty$ have finite trace; this proves the assertions (2.2) to (2.4). Eq. (2.5) follows from (2.2) to (2.4) since $\mathscr{H}$ has been assumed as infinite dimensional, and (2.6) is an obvious property of regular operators.

The characteristics (2.2) to (2.4) are equivalent to the property $\sigma_{\text {ess }}(A)=\emptyset$. According to Lemma 4 , a regular operator $A$ has a spectral representation $A=\sum_{n=1}^{\infty} a_{n} P_{a_{n}}$ with which the condition (2.1) can be put in the form

$$
\sum_{n=1}^{\infty} e^{-\beta a_{n}} \operatorname{Tr}\left(P_{a_{n}}\right)=\sum_{a_{i} \in S(A)} e^{-\beta a_{i}}<\infty .
$$

The following lemma shows that, on the basis of the entropy concept (1.11), QMEs exist only in the case that the operator $A$ in (1.12) has a pure point spectrum.

Lemma 5. If $A$ is a s. a. operator with $\sigma_{\text {ess }}(A) \neq \emptyset$ and $m$ is a real number with $\sigma(A)<m<\bar{\sigma}(A)$, then there always exist several elements $W^{\#} \in \mathfrak{S}_{A}{ }^{m}$ with the property $H\left(W^{\#}\right)=\infty$.

Proof. We first construct, for an arbitrary countably infinite orthonormal system $\left\{\varphi_{i}: i \in \mathrm{N}\right\}$ of $\mathscr{H}$, a state operator $\hat{W}$ with $\hat{W} \leqq \sum_{i=1}^{\infty} P\left(\varphi_{i}\right), H(\hat{W})=\infty$ and all the nonzero eigenvalues nondegenerate. For that purpose we introduce new double indices $r, k$ where the first index runs through the natural numbers while the second runs from 0 to $n_{r} \equiv 2^{r}-1$. This relabelling occurs according to the prescription

$$
\begin{aligned}
\varphi_{i} \triangleq \varphi_{r k} \Leftrightarrow i & =(k+1)+\sum_{j=1}^{r-1}\left(n_{j}+1\right) \\
& =k+\left(2^{r}-1\right)=n_{r}+k .
\end{aligned}
$$

By means of the coefficients

$$
c_{r k} \equiv d\left(2-k / n_{r}\right) / r^{2} 2^{r}, \quad d \equiv 4 / \pi^{2},
$$

we then construct the operator

$$
\hat{W} \equiv \sum_{r=1}^{\infty} \sum_{k=0}^{n_{r}} c_{r k} P\left(\varphi_{r k}\right)
$$

with the properties

$$
\begin{gathered}
(\forall r, k) c_{r k}>0, \\
(r, k) \neq\left(r^{\prime}, k^{\prime}\right) \Rightarrow c_{r k} \neq c_{r^{\prime} k^{\prime}}, \\
\sum_{k=0}^{n_{r}} c_{r k}=\frac{1}{2}\left(n_{r}+1\right)\left(c_{r 0}+c_{r n_{r}}\right)=3 d / 2 r^{2} \\
=6 / \pi^{2} r^{2}, \\
\operatorname{Tr}(\hat{W})=\sum_{r=1}^{\infty} \sum_{k=0}^{n_{r}} c_{r k}=\sum_{r=1}^{\infty} 6 / \pi^{2} r^{2}=1, \\
H(\hat{W})=\sum_{r=1}^{\infty} \sum_{k=0}^{n_{r}} c_{r k}\left|\ln c_{r k}\right| \geqq \sum_{r=1}^{\infty}\left|\ln c_{r 0}\right| \sum_{k=0}^{n_{r}} c_{r k} \\
\geqq\left(6 / \pi^{2}\right) \ln 2 \sum_{r=1}^{\infty} 1 / r=\infty .
\end{gathered}
$$

By changing the coefficients of $\hat{W}$ for finite index sets or by allowing of finite degeneracies, one can produce infinitely many state operators $\hat{W}$ with $\hat{W} \leqq \sum_{i=1}^{\infty} P\left(\varphi_{i}\right)$ and $H(\hat{W})=\infty$.

Next we consider two state operators $W_{1}, W_{2}$ with $W_{1} W_{2}=\mathrm{O}$. Accordingly,

$$
W_{3}=\gamma W_{1}+(1-\gamma) W_{2}
$$

is also a state operator for all $\gamma \in(0,1)$, and we find the relation

$$
\begin{aligned}
H\left(W_{3}\right)=\gamma H\left(W_{1}\right) & +(1-\gamma) H\left(W_{2}\right)+\gamma|\ln \gamma| \\
& +(1-\gamma|\ln (1-\gamma)| .
\end{aligned}
$$

Hence if a state operator $\hat{W}$ with $H(\hat{W})=\infty$ and an elementary projection operator $P(\chi)$ with $\hat{W} P(\chi)=\mathrm{O}$ are given, then all state operators $W^{\#}=\gamma P(\chi)+(1-\gamma) \hat{W}$ with $0<\gamma<1$ have the property $H\left(W^{\#}\right)=\infty$.

With these preliminaries we can prove the statement of Lemma 5. Let $A$ be a s.a. operator with $\sigma_{\text {ess }}(A) \neq \emptyset$. First we consider the case that $\sigma_{\text {ess }}(A)$ contains an element $z \neq m$; without loss of generality we can assume $m<z$. Because $\sigma(A)<m$, there exists at least one element $y \in \bar{\sigma}(A)$ with $y<m$ and hence we can choose two positive, real numbers $\eta, \varepsilon$ with the properties

$$
\begin{gathered}
y+\eta<m<z-\varepsilon, \\
\max (2 \eta, 2 \varepsilon)<\min (z-m, m-y), \\
\operatorname{Tr}\left(R_{z^{\varepsilon}}\right)=\infty, \quad \operatorname{Tr}\left(R_{y} \eta\right) \geqq 1
\end{gathered}
$$


where we have introduced the notation

$$
R_{y}{ }^{\alpha} \equiv E_{A}(x+\alpha)-E_{A}(x-\alpha) .
$$

To every pair of numbers $u, v$ with

$$
y-\eta \leqq u \leqq y+\eta, \quad z-\varepsilon \leqq v \leqq z+\varepsilon
$$

we associate the number $\gamma_{u v} \equiv(v-m) /(v-u)$ which has the properties

$$
0<\gamma_{u v}<1, \quad m=\gamma_{u v} u+\left(1-\gamma_{u v}\right) v .
$$

By choosing an arbitrary state operator $\hat{W}$ with $H(\hat{W})=\infty, \hat{W} \leqq R_{z} \varepsilon$, as constructed in (2.11), and an arbitrary unit element $\chi \in R_{y} \eta \mathscr{H}$, we find

$$
\begin{gathered}
c \equiv \operatorname{Tr}(\hat{W} A)=\int_{z-\varepsilon}^{z+\varepsilon} \lambda \mathrm{d} \operatorname{Tr}\left[\hat{W} E_{A}(\lambda)\right] \in[z-\varepsilon, z+\varepsilon], \\
b \equiv \operatorname{Tr}(P(\chi) A) \\
\quad=\int_{y-\eta}^{y+\eta} \lambda \mathrm{d}\left(\chi, E_{A}(\lambda) \chi\right) \in[y-\eta, y+\eta] .
\end{gathered}
$$

If we now construct the s.a. operator

$$
W^{\#} \equiv \gamma_{b c} P(\chi)+\left(1-\gamma_{b c}\right) \hat{W},
$$

then the Eqs. (2.13), (2.15) and (2.16) yield

$$
\begin{aligned}
\operatorname{Tr}\left(W^{\#}\right) & =\gamma_{b c}+\left(1-\gamma_{b c}\right) \operatorname{Tr}(\hat{W})=1, \\
H\left(W^{\#}\right) & \geqq\left(1-\gamma_{b c}\right) H(\hat{W})=\infty, \\
\operatorname{Tr}\left(W^{\#} A\right) & =\gamma_{b c} b+\left(1-\gamma_{b c}\right) c=m .
\end{aligned}
$$

Finally there remains the possibility $\sigma_{\text {ess }}(A)$ $=\{m\}$. In this case, $m$ is either an eigenvalue of infinite multiplicity or a limit point of the eigenvalues of $A$. If $m$ is an eigenvalue with $\operatorname{Tr}\left(P_{m}\right)=\infty$, then every state operator $\hat{W}$ with $\hat{W} \leqq P_{m}$, $H(\hat{W})=\infty$ already has the desired properties. If $m$ is a limit point of the point spectrum of $A$, then at least one of the two intervals $(m-\delta, m)$ and $(m, m+\delta), \delta>0$, includes infinitely many eigenvalues of $A$. Let this be the case with $(m, m+\delta)$; then we have $\operatorname{Tr}\left\{E_{A}(m+\delta)-E_{A}(m)\right\}=\infty$. Because $\sigma(A)<m$ and $\sigma_{\mathrm{ess}}(A)=\{m\}$, there exists also an eigenvalue $b$ with $b<m$. If we now choose a unit element $\chi \in P_{b} \mathscr{H}$ and a state operator $\hat{W}$ with $\hat{W} \leqq E_{A}(m+\delta)-E_{A}(m)$ and $H(\hat{W})=\infty$, then we arrive, just as above, at a state operator $W^{\#}$ with the properties (2.17). And since many state operators $W^{\#}$ with the properties $(2.17)$ can be constructed in this way the proof of the lemma is completed.

With the aid of the above lemmata one easily infers the following theorem.
Theorem I. a) Let $A$ be a s.a. operator and $m$ be a real number with $\sigma(A)<m<\bar{\sigma}(A)$. Then $\sup \left\{H(W): W \in \mathfrak{S}_{A}{ }^{m}\right\}$ is finite if and only if $A$ is regular.

b) If $A$ is a s.a. operator with $\sigma_{\mathrm{ess}}(A)=\emptyset$ and $\bar{\sigma}(A)<\infty$, then $\bar{\sigma}(A)$ is an eigenvalue of $A$ and we have

$$
\sup \left\{H(W): W \in \mathfrak{W}_{A}{ }^{\bar{\sigma}}\right\}=\ln \operatorname{Tr}\left(P_{\bar{\sigma}}\right)<\infty .
$$

The same holds for a finite $\underline{\sigma}(A)$.

c) If $A$ is $a$ s.a. operator with $\sigma_{\mathrm{ess}}(A) \neq \emptyset$ and $\bar{\sigma}(A)<\infty$, then $\mathfrak{S}_{A} \overline{\bar{\sigma}}$ is non-empty if and only if $\bar{\sigma}(A)$ is an eigenvalue of $A$. In this case we have $\sup \left\{H(W): W \in \mathfrak{S}_{A} \bar{\sigma}\right\}=\ln \operatorname{Tr}\left(P_{\bar{\sigma}}\right)$. The same holds for a finite $\underline{\sigma}(A)$.

Proof. ad a) Let $A$ be regular; then the essential spectrum of $A$ is empty and $A$ has a countable spectral set $S(A)=\left\{a_{i}: i \in \mathbf{N}\right\}$. According to Corollary 2 one can then restrict oneself, in search of the supremum of $H(W)$ on $\mathfrak{S}_{A}{ }^{m}$, to state operators of the form $W=\sum_{i=1}^{\infty} w_{i} P\left(\varphi_{i}\right)$ where $\left\{\varphi_{i}: i \in \mathrm{N}\right\}$ is an $A$-basis whose elements are labelled in line with the corresponding elements of $S(A)$. Hence it follows that $\operatorname{Tr}(W A)=\sum_{i=1}^{\infty} a_{i} w_{i}$, and Lemma 3 proves the assertion $\sup \left\{H(W): W \in \mathfrak{S}_{A}{ }^{m}\right\}<\infty$.

If we start from the assumption

$$
\sup \left\{H(W): W \in \mathfrak{S}_{A} m\right\}<\infty,
$$

then Lemma 5 yields $\sigma_{\mathrm{ess}}(A)=\emptyset$ and, consequently, Lemma 3 and Corollary 2 prove the existence of a real number $\beta$ with

$$
\sum_{a_{i} \in S(A)} e^{-\beta a_{i}}=\operatorname{Tr}\left(e^{-\beta A}\right)<\infty .
$$

Thus $A$ is regular.

$a d b)$ In case of $\sigma_{\text {ess }}(A)=\emptyset$ and $\bar{\sigma}(A)<\infty$, $\bar{\sigma}$ is an eigenvalue of (at most) finite multiplicity since $\sigma(A)$ is closed. Hence all $W \in \mathfrak{S}_{A}{ }^{\bar{\sigma}}$ satisfy $W \leqq P_{\bar{\sigma}}$ and Eq. (2.18) results from the obvious fact that the set of all state operators less than or equal to a finite dimensional projection operator $Q$ includes a unique $\mathrm{QME}$, namely $\bar{W}=Q / \operatorname{Tr}(Q)$.

ad c) By inspection one can see that the equation

$$
\infty>\bar{\sigma}(A)=\operatorname{Tr}(W A)=\int_{-\infty}^{\bar{\sigma}} \lambda \mathrm{d} \operatorname{Tr}\left[W E_{A}(\lambda)\right]
$$


has a solution $W$ if and only if $\bar{\sigma}(A)$ is an eigenvalue of $A$ and $W \leqq P_{\bar{\sigma}}$. In this case the assertion follows from (2.18).

Corollary 6. If $A$ is regular and if $\sigma(A) \leqq m \leqq$ $\bar{\sigma}(A)$, then $\sup \left\{H(W): W \in \mathfrak{L}_{A} m\right\}<\bar{\infty}$.

While stating the conditions for

$$
\sup \left\{H(W): W \in \mathfrak{L}_{A}{ }^{m}\right\}<\infty,
$$

Theorem I does not specify in which cases this finite supremum is in fact assumed and whether the eventual maximum is unique. These questions are answered by Theorem II.

According to Lemma 4 all regular operators are bounded from exactly one side. Without loss of generality we will confine ourselves in the following to regular operators which are bounded from below ; regular operators bounded from above can be treated in complete analogy. For that reason we can presume the following properties of $S(A)$ :

$$
\begin{gathered}
S(A)=\left\{a_{1}, a_{2}, \ldots\right\}, \\
-\infty<a_{1} \leqq a_{2} \leqq a_{3} \leqq \ldots, \\
\lim _{i \rightarrow \infty} a_{i}=\infty, \quad\left(\exists N_{0} \in \mathrm{N}\right)\left(\forall n>N_{0}\right) a_{n}>0, \\
\left(\forall n \leqq N_{0}\right) a_{n} \leqq 0 .
\end{gathered}
$$

Next we introduce some notations to facilitate the formulation of Theorem II. We set

$$
\begin{gathered}
Z_{A}(\beta) \equiv \operatorname{Tr}[\exp (-\beta A)]=\sum_{i=1}^{\infty} e^{-\beta a_{i}}, \\
\hat{\beta}_{A} \equiv \inf \left\{\beta \in \mathrm{R}: Z_{A}(\beta)<\infty\right\} .
\end{gathered}
$$

Obviously, $\hat{\beta}_{A} \geqq 0$. If $Z_{A}\left(\hat{\beta}_{A}\right)<\infty$, then $\hat{\beta}_{A}$ is called critical. In this case we define

$$
\hat{m}_{A} \equiv Z_{A}\left(\hat{\beta}_{A}\right)^{-1} \operatorname{Tr}\left[A \cdot \exp \left\{-\hat{\beta_{A}} A\right\}\right] .
$$

It should be noted that $\hat{m}_{A}$ is not necessarily finite. If $\hat{\beta}_{A}$ is not critical, i.e. if $Z_{A}\left(\hat{\beta}_{A}\right)=\infty$, then we set $\hat{m}_{A}=\infty$. Finally we introduce for every $\beta \in\left(\hat{\beta}_{A}, \infty\right)-$ and also for $\hat{\beta}_{A}$ if $\hat{\beta}_{A}$ is critical the s.a. operator

$$
V_{A}(\beta) \equiv Z_{A}(\beta)^{-1} \exp \{-\beta A\} .
$$

For simplicity we will often drop the index $A$ if no confusion results.

Theorem II. Let $A$ be a regular operator bounded from below. Then we find:

a) For all $\beta \in(\hat{\beta}, \infty)$ the operator $V(\beta)$ is a state operator. b) The correspondence $\beta \mapsto\langle A\rangle(\beta) \equiv \operatorname{Tr}[V(\beta) A]$ maps the interval $(\hat{\beta}, \infty)$ one-to-one and continuously onto the interval $\left(a_{1}, \hat{m}\right)$.

For $m=a_{1}, H$ assumes its maximum $\ln \operatorname{Tr}\left(P_{a_{1}}\right)$ at the QME $P_{a_{1}} / \operatorname{Tr}\left(P_{a_{1}}\right)$. For $m=\hat{m}, H$ assumes its supremum on $\mathfrak{S}_{A}{ }^{m}$ at $\hat{W}[\hat{m}]$ if and only if $\hat{m}$ is finite. For $\hat{m}<m<\infty, H$ never assumes its supremum on $\mathfrak{S}_{A} m$.

c) For $a_{1}<m<\hat{m}$ one obtains

$$
\begin{aligned}
H(\hat{W}[m]) & =\sup \left\{H(W): W \in \mathfrak{I}_{A} m\right\} \\
& =\ln Z\left(\langle A\rangle^{-1}(m)\right)+m\langle A\rangle^{-1}(m) ;
\end{aligned}
$$

for $\hat{m} \leqq m<\infty$ one obtains

$$
\sup \left\{H(W): W \in \mathfrak{S}_{A} m\right\}=\ln Z(\hat{\beta})+m \hat{\beta} .
$$

d) In the interval $(\hat{\beta}, \infty)$ the functions $Z,\langle A\rangle$ and $H[V(\cdot)]$ are differentiable and yield

$$
\begin{aligned}
\langle A\rangle(\beta) & =-\mathrm{d} \ln Z(\beta) / \mathrm{d} \beta, \\
\mathrm{d}\langle A\rangle(\beta) / \mathrm{d} \beta & =-\mathrm{d}^{2} \ln Z(\beta) / \mathrm{d} \beta^{2} \\
& =-\left\langle(A-\langle A\rangle)^{2}\right\rangle<0, \\
\mathrm{~d} H[V(\beta)] / \mathrm{d} \beta & =\beta \mathrm{d}\langle A\rangle / \mathrm{d} \beta<0 .
\end{aligned}
$$

The function $H(\hat{W}[\cdot])$ is also differentiable in the interval $\left(a_{1}, \hat{m}\right)$ and yields

$$
\left.\mathrm{d} H(\hat{W}[m]) / \mathrm{d} m=\langle A\rangle^{-1}(m)=\beta\right\rangle 0 .
$$

If $A$ is a regular operator bounded from above, then analogous statements hold true with negative $\beta$ and $\hat{\beta} \equiv \sup \{\beta: Z(\beta)<\infty\} \leqq 0$.

e) For every $m \in\left(a_{1}, \hat{m}\right)$, the information entropy $H$ has a unique maximum on the set $\mathfrak{S}_{A} m$ which is assumed at the QME $\hat{W}[m] \equiv V\left(\langle A\rangle^{-1}(m)\right)$ :

$$
\begin{gathered}
\left(\forall m \in\left(a_{1}, \hat{m}\right)\right)\left(\forall W \in \mathfrak{S}_{A}{ }^{m}\right) \\
W \neq \hat{W}[m]) \Leftrightarrow H(W)<H(\hat{W}[m])<\infty .
\end{gathered}
$$

With the aid of Corollary 2, the proof of all assertions of this theorem can be found in Reference ${ }^{2}$.

The Theorems I and II completely characterize the conditions under which the given information " $\operatorname{Tr}(W A)=m, m \in \mathrm{R}$ " determines a unique QME: A QME exists if and only if either $A$ is regular and ${ }^{17} m \in\left(a_{1}, \hat{m}_{A}\right]\left[m \in\left[\hat{m}_{A}, a_{1}\right)\right]$ or $m$ is equal to $\bar{\sigma}(A)$ or $\sigma(A)$ and is coincidently an eigenvalue of $A$ with finite multiplicity. If, on the other hand, $A$ is regular but $\hat{m}_{A}<m\left[\hat{m}_{A}>m\right]$ then the supremum of $H$ on $\mathfrak{S}_{A} m$ is still finite but "unattainable", i.e. $\mathfrak{B}_{A}{ }^{m}$ contains no QME even though

$$
\sup \left\{H(W): W \in \mathfrak{S}_{A} m\right\}<\infty .
$$


Remark. As is shown by the above results, either only positive or negative " $A$-temperatures" $1 / \beta$ can occur in q.m. systems with an infinite dimensional Hilbert space - depending on whether $A$ is bounded from below or above. Moreover, Theorem II includes the remarkable possibility of an upper limit $|\mathbf{1}| \hat{\beta}_{A} \mid$ of the absolute value of the $A$-temperature. In the special case $Z_{A}(\hat{\beta})<\infty,\left|\hat{m}_{A}\right|<\infty$, there even exists a $\mathrm{QME}$ with the maximum [minimum] $A$-temperature $\hat{\beta}_{A}^{-1}$, namely the state operator $V_{A}(\hat{\beta})$. In contrast to this, q.m. systems with a finite dimensional Hilbert space can have positive as well as negative $A$-temperatures ${ }^{18}$. But in such systems there exists no upper limit of $\left|\beta^{-1}\right|$.

Definition 2. A s.a. operator $A$ is called weakly irregular (w.ir.) if $\sigma_{\mathrm{ess}}(A)=\emptyset$ but if $A$ is not regular.

The w.ir. operators are divided into two classes: the w.ir. operators bounded from one side and the w.ir. operators bounded from no side. As is shown by the following lemmata, both classes contain operators which allow an infinite supremum of $H$ on $\mathfrak{W}_{A}{ }^{m}$ as well as operators admitting only a finite supremum of $H$ on $\mathfrak{S}_{A}{ }^{m}$. First we consider the w.ir. operators bounded from one side; without loss of generality, we can confine ourselves to operators which are bounded from below and whose spectral set has the properties (2.19).

Lemma 7. Let $A$ be a s.a. operator bounded from below with $\sigma_{\text {ess }}(A)=\emptyset$ and let $m$ be a real number with $m>a_{1}$. Then:

1) If there exist numbers $N_{1} \in \mathrm{N}, 0<p<\infty$, $0<\varepsilon<1$ such that the elements of $S(A)$ satisfy the condition

$$
\left(\forall k \geqq N_{1}\right) a_{k} \leqq p(\ln k)^{1-\varepsilon},
$$

then $A$ is w.ir. and there exist several state operators $W^{\#} \in \mathfrak{S}_{A}{ }^{m}$ with $H\left(W^{\#}\right)=\infty$.

2) If, on the other hand, there exist numbers $N_{2} \in \mathrm{N}, 0<q<\infty$ such that the elements of $S(A)$ satisfy the condition

$$
\left(\forall k \geqq N_{2}\right) a_{k} \geqq q \ln k,
$$

then $A$ is regular and consequently

is finite.

$$
\sup \left\{H(W): W \in \mathfrak{S}_{A}{ }^{m}\right\}
$$

Proof. ad 1) If $S(A)=\left\{a_{1}, a_{2}, \ldots\right\}$ is the monotonically increasing spectral set of $A,\left\{\varphi_{i}: i \in \mathbf{N}\right\}$ a corresponding $A$-basis, $N_{0}$ the index of (2.19) and
$K \equiv \max \left(N_{0}, N_{1}\right)$, then we obtain

$$
\begin{aligned}
\operatorname{tr}_{\varphi}\left[e^{-c A}\right] & \equiv \sum_{i=1}^{\infty}\left(\varphi_{i}, e^{-c A} \varphi_{i}\right) \\
& \geqq \sum_{i=K}^{\infty} e^{-c a_{i}} \geqq \sum_{i=K}^{\infty} \exp \left\{-c p(\ln i)^{1-\varepsilon}\right\}
\end{aligned}
$$

for all $c>0$. With given numbers $p>0,0<\varepsilon<1$, there exists for every $c>0$ a smallest natural number $\tilde{N}_{c}$ with the property

$$
\left(\forall k \geqq \tilde{N}_{c}\right) \quad \ln k \geqq c p(\ln k)^{1-\varepsilon} .
$$

Setting $N_{c} \equiv \max \left(K, \tilde{N}_{c}\right)$ we obtain

$$
\begin{aligned}
\operatorname{tr}_{\varphi}\left[e^{-c A}\right] & \geqq \sum_{i=N_{c}}^{\infty} \exp \left\{-c p(\ln i)^{1-\varepsilon}\right\} \\
& \geqq \sum_{i=N_{c}}^{\infty} \exp \{-\ln i\}=\sum_{i=N_{c}}^{\infty} 1 / i=\infty .
\end{aligned}
$$

Hence if $A$ satisfies the conditions (2.22) and $\sigma_{\text {ess }}(A)=\emptyset$, then $A$ is w.ir.

Let $A=\sum_{i=1}^{\infty} a_{i} P\left(\varphi_{i}\right)$ be the diagonal representation corresponding to the $A$-basis $\left\{\varphi_{i}: i \in \mathrm{N}\right\}$. Temporarily we pass over to the double indices of (2.9) and consider the state operator

which yields

$$
\hat{W} \equiv \sum_{r=1}^{\infty} \sum_{k=0}^{n_{r}} c_{r k} P\left(\varphi_{r k}\right)
$$

$$
\begin{aligned}
\operatorname{Tr}(\hat{W} A) & =\sum_{r=1}^{\infty} \sum_{k=0}^{n_{r}} a_{r k} c_{r k} \\
& \leqq \sum_{r=1}^{\infty} n_{r} \cdot \max a_{r k} \cdot \max c_{r k} .
\end{aligned}
$$

With $\max c_{r k}=c_{r 0}=2 d / r^{2} 2^{r}$,

$$
0 \leq k \leq n_{r}
$$

$$
\begin{aligned}
\max _{0 \leq k \leq n_{r}} a_{r k} & \leqq a_{r+1,0} \leqq p\left\{\ln \left(2^{r+1}-1\right)\right\}^{1-\varepsilon} \\
& \leqq p[(r+1) \ln 2]^{1-\varepsilon}
\end{aligned}
$$

and $n_{r}=2^{r}-1$ we finally obtain

$$
\begin{aligned}
\operatorname{Tr}(\hat{W} A) \leqq & \sum_{r=1}^{\infty}\left(2^{r}-1\right)\left(2 d / r^{2} 2^{r}\right) p(\ln 2)^{1-\varepsilon}(r+1)^{1-\varepsilon} \\
& \leqq 4 p \sum_{r=1}^{\infty}(r+1)^{1-\varepsilon} / r^{2}<\infty
\end{aligned}
$$

for $\varepsilon>0$. If $\operatorname{Tr}(\hat{W} A)$ is equal to $m$, then $\hat{W}$ itself represents an element of $\mathfrak{S}_{A}{ }^{m}$ with $H(\hat{W})=\infty$. In the general case of $\operatorname{Tr}(\hat{W} A) \neq m$, say $\operatorname{Tr}(\hat{W} A)$ $\equiv x>m>a_{1}$, we construct an "adjusted" state operator

$$
W^{\#} \equiv \gamma P\left(\varphi_{1}\right)+(1-\gamma) \hat{W},
$$


$\gamma=(x-m) /\left(x-a_{1}\right)$ with the desired properties $\operatorname{Tr}\left(W^{\#} A\right)=m$ and $H\left(W^{\#}\right)=\infty$. Obviously one can find many such state operators.

$$
\begin{aligned}
\text { ad 2) Setting } M & \equiv \max \left(N_{0}, N_{2}\right) \text { and } \\
C & \equiv \sum_{i=1}^{M-1} \exp \left\{-2 a_{i} / q\right\}
\end{aligned}
$$

we obtain $0 \leqq C<\infty$ and

$$
\begin{aligned}
\operatorname{Tr}\{\exp (-2 A / q)\} & =\sum_{i=1}^{\infty} \exp \left\{-2 a_{i} / q\right\} \\
& \leqq C+\sum_{i=M}^{\infty} e^{-2 \ln i} \\
& \leqq C+\sum_{i=M}^{\infty} 1 / i^{2}<\infty
\end{aligned}
$$

and accordingly $A$ is regular.

Finally we consider the class of all w.ir. operators which are unbounded on both sides. The spectral set $S(A)=\left\{a_{1}, a_{2}, \ldots\right\}$ of these operators can be arranged such as to have the properties

$$
\begin{aligned}
& \ldots \leqq a_{4} \leqq a_{2}<0 \leqq a_{1} \leqq a_{3} \leqq \ldots \\
& \lim _{n \rightarrow \infty} a_{2 n}=-\infty, \quad \lim _{n \rightarrow \infty} a_{2 n+1}=\infty .
\end{aligned}
$$

Lemma 8. Let $A$ be a w.ir. operator with the spectral set $(2.24)$ and let $m$ be an arbitrary real number. Then:

1) If there exist numbers $N_{1} \in \mathrm{N}$ and $0<\varepsilon<\infty$, $0<p<\infty$ with the property

$$
\left(\forall k \geqq N_{1}\right)\left|a_{k}\right| \geqq p k^{1+\varepsilon},
$$

then $H(W)$ is finite on $\mathfrak{L}_{A}{ }^{m}$ even though

$$
\sup \left\{H(W): W \in \mathfrak{B}_{A}^{m}\right\}=\infty .
$$

2) If, on the other hand, there exist numbers $N_{2} \in \mathrm{N}, 0<q<\infty$ with the property

$$
\left(\forall k \geqq N_{2}\right)\left|a_{k}\right| \leqq q k,
$$

then $\mathfrak{S}_{A} m$ contains several elements $W^{\#}$ with $H\left(W^{\#}\right)=\infty$.

Proof. ad 1) Since we are interested only in state operators with the greatest possible information entropy we can, according to Corollary 2, confine ourselves to state operators of the form

$$
W=\sum_{i=1}^{\infty} w_{i} P\left(\varphi_{i}\right)
$$

where $\left\{\varphi_{i}: i \in \mathrm{N}\right\}$ is an $A$-basis, whose elements are labelled in accord with the elements of $S(A)$ in (2.24). For these state operators we obtain

$$
\operatorname{Tr}(W A)=\sum_{i=1}^{\infty} w_{i} a_{i}
$$

Since the $w_{i} a_{i}$ form an alternating sequence the sum in (2.27) exists if and only if the $w_{i} a_{i}$ tend to

zero. Hence the assumptions $(2.25)$ and $\sum_{i=1}^{\infty} w_{i} a_{i}<\infty$ yield the (rough) estimate

$\left(\exists N_{3} ; 3 \leqq N_{3}<\infty\right)\left(\forall k \geqq N_{3}\right) w_{k} \leqq\left(p k^{1+\varepsilon}\right)^{-1}$

and we obtain

$H(W) \leqq N_{3}+(1 / p) \sum_{k=N_{3}}^{\infty}[\ln p+(1+\varepsilon) \ln k] / k^{1+\varepsilon}$ $<\infty$.

ad 2) Let $\left\{\varphi_{i}: i \in \mathbf{N}\right\}$ be an $A$-basis whose elements are labelled in accord with the elements of $S(A)$ in (2.24). Again we temporarily pass over to the double indices of $(2.9)$ and consider the state operator

$$
\hat{W} \equiv \sum_{r=1}^{\infty} \sum_{k=0}^{n_{r}} c_{r k} P\left(\varphi_{r k}\right) .
$$

From the Eqs. (2.26) and (2.10) it follows then

$$
\begin{aligned}
\left|a_{r k} c_{r k}\right| \leqq q\left(k+2^{r}-1\right) \frac{d}{2^{r} r^{2}}\left(2-\frac{k}{2^{r}-1}\right) \\
\leqq \frac{4 q}{r^{2}} \frac{2^{r}-1}{2^{r}}
\end{aligned}
$$

for all $(r, k) \triangleq i \geqq N_{2}$.

Let us now consider the expression

$$
\operatorname{Tr}(\hat{W} A)=\sum_{r=1}^{\infty} \sum_{k=0}^{n_{r}} c_{r k} a_{r k}=\sum_{i=1}^{\infty} c_{i} a_{i}
$$

in the second sum of which we have returned to the single index. By reason of the structure of $S(A)$, the $c_{i} a_{i}$ form an alternating sequence and according to $(2.28)$ they also tend to zero. Hence $\operatorname{Tr}(\hat{W} A)$ is finite and just as in the proof of Lemma 7 one can construct several state operators $W$ \# with the properties $W^{\#} \in \mathfrak{S}_{A} m$ and $H\left(W^{\#}\right)=\infty$.

Unfortunately, the criteria (2.22), (2.23), (2.25) and $(2.26)$ do not enable the complete decompsition of the set of all w.ir. operators into the two classes mentioned above. 


\section{Conclusion}

The information " $M(A)=\operatorname{Tr}(W A)=m, m$ finite" determines a unique quantum state with maximum information entropy if and only if one of the following conditions is fulfilled

(a) $A$ is thermodynamically regular and $m$ satisfies the equation $\underline{\sigma}(A)<m \leqq \hat{m}_{A}$ or $\bar{\sigma}(A)>m \geqq \hat{m}_{A}$

1 E. T. Jaynes, Phys. Rev. 106, 620 [1957]; 108, 171 [1957].

2 R. S. Ingarden and K. Urbanik, Acta Phys. Polon. 21, 281 [1962].

3 E. T. Jaynes, Information Theory and Statistical Physics, in: 1962 Brandeis Lectures, Vol. 3, Benjamin, New York 1963.

4 For further references to this subject we refer to a recent monograph: A. Hobson, Concepts in Statistical Mechanics, Gordon and Breach, New York 1971.

5 J. v. Neumann, Mathematische Grundlagen der Quantenmechanik, Springer-Verlag, Berlin 1932.

${ }^{6}$ G. Ludwig, Die Grundlagen der Quantenmechanik, Springer-Verlag, Berlin 1954.

7 J. Langerholc, J. Math. Phys. 6, 1210 [1965].

8 For simplicity we denote q.m. observables and quantum states by the same symbol as the respective representing s. a. operators and state operators.

9 U. Fano, Rev. Mod. Phys. 29, 74 [1957]. depending on whether $A$ is bounded from below or above.

(b) $m$ equals $\bar{\sigma}(A)$ or $\sigma(A)$ and is coincidently an eigenvalue of $A$ with finite multiplicity.

The generalisation of this result to the case in which the mean values of several quantities are given will be treated in a subsequent paper.

10 This formula was first derived by v. Neumann ${ }^{11}$ for the thermodynamic entropy of a q.m. system in thermal equilibrium by means of thermodynamic arguments.

11 J. v. Neumann, Gött. Nachr. 1, 273 [1927].

12 A. Stahl, Z. Naturforsch. 15 a, 655 [1960].

13 R. S. Ingarden and A. Kossakowski, Bull. Acad. Polon. Sci., Ser. Sci. Math. 16, 61 [1968].

14 F. Engelmann, M. Feix, E. Minardi, and J. Oxenius, Z. Naturforsch. 16 a, 1223 [1961].

15 A comparison of these two entropy concepts is found in 12,13 and in: H. Schwegler, Z. Naturforsch. 20 a, 1543 [1965] ; F. Schlögl, A. Stahl, and R. Bausch, Z. Phys. 187, 290 [1965].

16 E. H. Wichmann, J. Math. Phys. 4, 884 [1963].

17 Whereas the normal text refers to a regular operator bounded from below, the insertions in square brackets refer to an $A$ bounded from above.

18 F. N. Ramsey, Phys. Rev. 103, 20 [1956]. - Y. P. Terletskii, Statistical Physics, North-Holland, Amsterdam 1971.

\title{
Coherent States and the Magnetic Operators
}

\author{
A. D. Jannussis and L. Papaloucas \\ Department of Theoretical Physics, University of Patras, Greece
}

(Z. Naturforsch. 28 a, 701-704 [1973] ; received 27 December 1972)

\begin{abstract}
In the present paper a method of calculation of the density matrix in the Schrödinger picture is given, in terms of the known creation and annihiliation operators $a^{+}$and $a$. New magnetic operators are given with the help of the coherent states, which depend on two free parameters $\mu$ and $\nu$. For the case $\mu=v=(e H) /(2 \hbar c)$, these operators lead to the well-known magnetic operators, as they are given in the current litterature.
\end{abstract}

\section{$\S 1$. Introduction}

The physical significance of the coherent states is very important. They are, not only the states of oscillations that we encounter in nature, but also the states which are produced when an oscillator is coupled linearly with a prescribed classical force. They are also emitted by a classical current source. Therefore, they have many significant applications.

Reprint requests to Prof. Dr. A. D. Jannussis, Department of Theoretical Physics, University of Patras, Patras/Griechenland.
The coherent states were first introduced by Glauber $^{1}$, as eigenkets of the lowering operator $a$, defined by the equation:

$$
a|\alpha\rangle=\alpha|\alpha\rangle \text {. }
$$

Applications of the coherent states to the density operator of boson amplitude operators, have been given by Cahill and Glauber ${ }^{2}$. Also by Grosiqueni and Solimento ${ }^{3}$, to the master equation for the $p$ representation in the Schrödinger picture.

The equation of motion of the density matrix in the Schrödinger picture, is of the form:

$$
i \hbar \cdot \partial \varrho / \partial t=\{\mathcal{H}, \varrho\} .
$$

